PROCEEDINGS OF THE

AMERICAN MATHEMATICAL SOCIETY

Volume 131, Number 1, Pages 45-53

S 0002-9939(02)06526-7

Article electronically published on May 15, 2002

\title{
ON SOME PROPERTIES OF (FC)-SEQUENCES OF IDEALS IN LOCAL RINGS
}

\author{
DUONG QUÔC VIỆT
}

(Communicated by Wolmer V. Vasconcelos)

\begin{abstract}
The paper characterizes the length of maximal sequences satisfying conditions (i) and (ii) of (FC)-sequences, and proves some properties of (FC)-sequences, such as a bound on their lengths. As a consequence we get some results for mixed multiplicities and multiplicities of Rees rings of equimultiple ideals. We also prove that if $I$ is an ideal of positive height and $x_{1}, x_{2}, \ldots, x_{p}$ is an arbitrary maximal sequence in $I$ satisfying conditions (i) and (ii) of (FC)-sequences, then $\left(x_{1}, x_{2}, \ldots, x_{p}\right)$ is a reduction of $I$.
\end{abstract}

\section{INTRODUCTION}

Throughout this paper, let $(A, \mathfrak{m})$ be a Noetherian local ring with maximal ideal $\mathfrak{m}$, infinite residue field $k=A / \mathfrak{m}$, and Krull dimension $\operatorname{dim} A=d>0$. Let $J$ be an $\mathfrak{m}$-primary ideal and $\left\{I_{1}, I_{2}, \ldots, I_{s}\right\}$ a set of ideals of positive height. Then the Bhattacharya function

$$
B\left(n, n_{1}, \ldots, n_{s}\right)=l_{A}\left(\frac{J^{n} I_{1}^{n_{1}} \cdots I_{s}^{n_{s}}}{J^{n+1} I_{1}^{n_{1}} \cdots I_{s}^{n_{s}}}\right)
$$

is a polynomial of degree $d-1$ for all large values of $n, n_{1}, \ldots, n_{s}$. This polynomial is called the Bhattacharya polynomial of $\left(J, I_{1}, \ldots, I_{s}\right)$. The terms of total degree $d-1$ in this polynomial have the form

$$
\sum_{d_{0}+d_{1}+\cdots+d_{s}=d-1} e_{A}\left(J^{\left[d_{0}+1\right]}, I_{1}{ }^{\left[d_{1}\right]}, \ldots, I_{s}{ }^{\left[d_{s}\right]}\right) \frac{n^{d_{0}} n_{1} d_{1} \ldots n_{s} d_{s}}{d_{0} ! d_{1} ! \cdots d_{s} !} .
$$

Here $e_{A}\left(J^{\left[d_{0}+1\right]}, I_{1}{ }^{\left[d_{1}\right]}, \ldots, I_{s}{ }^{\left[d_{s}\right]}\right)$ are non-negative integers not all zero, called the mixed multiplicity of $\left(J, I_{1}, \ldots, I_{s}\right)$ of the type $\left(d_{0}+1, d_{1}, \ldots, d_{s}\right)[3]$.

Mixed multiplicities were first considered in [1] by Teissier and Risler for two $\mathfrak{m}$-primary ideals and in this case they can be interpreted as the multiplicity of general elements. Next, Rees in [9] showed the existence of joint reductions of $d$ $\mathfrak{m}$-primary ideals and the mixed multiplicity of those ideals is the multiplicity of the ideal generated by a joint reduction. A natural question is the relationship between mixed multiplicities of arbitrary ideals and Zariski-Samuel multiplicities. In answer to this question, Viet in [16] built a sequence of elements called an (FC)-sequence.

Received by the editors April 9, 2001 and, in revised form, August 16, 2001.

2000 Mathematics Subject Classification. Primary 13A15; Secondary 13H15.

Key words and phrases. (FC)-sequence, mixed multiplicity, multiplicity of Rees ring, reduction, equimultiple ideal.

The author was partially supported by the National Basic Research Program. 
Definition ([16]). Let $U=\left\{I_{1}, I_{2}, \ldots, I_{s}\right\}$ be a set of ideals of $A, I=I_{1} I_{2} \cdots I_{s}$, $N:=\bigcup_{n \geq 0}\left(0: I^{n}\right), A^{*}=A / N, I_{i}{ }^{*}=I_{i} A^{*} ; i=1,2, \ldots, s$. Recall that an element $x \in A$ satisfies the condition (FC) with respect to $U$ if there exist an ideal $I_{i}$ of $U$ and an integer $n_{i}^{\prime}$ such that

(i) $x \in I_{i} \backslash \mathfrak{m} I_{i}$ and

$$
I_{1}^{* n_{1}} \cdots I_{i}^{* n_{i}} \cdots I_{s}^{* n_{s}} \cap\left(x^{*}\right)=I_{1}^{* n_{1}} \cdots I_{i-1}^{*}{ }^{n_{i-1}} I_{i}^{* n_{i}-1} I_{i+1}^{*}{ }^{n_{i+1}} \cdots I_{s}^{* n_{s}} x^{*}
$$

for all $n_{i} \geq n_{i}^{\prime}$ and for all non-negative integers $n_{1}, \ldots, n_{i-1}, n_{i+1}, \ldots, n_{s}$, where $x^{*}$ is the image of $x$ in $A^{*}$.

(ii) $x$ is a filter-regular element with respect to $I$, i.e., $0: x \subseteq \bigcup_{n \geq 0}\left(0: I^{n}\right)$ (see [12]).

(iii) $\operatorname{dim} A / \bigcup_{n \geq 0}\left[(x): I^{n}\right]=\operatorname{dim} A / N-1$.

A sequence $x_{1}, x_{2}, \ldots, x_{t}$ of $A$ is said to be an (FC)-sequence with respect to $U$ if $\bar{x}_{i+1}$ is an element satisfying the condition (FC) with respect to $\bar{U}=\left\{\bar{I}_{1}, \bar{I}_{2}, \ldots, \bar{I}_{s}\right\}$ for each $i=0,1, \ldots, t-1$, where $\bar{A}=A /\left(x_{1}, x_{2}, \ldots, x_{i}\right), \bar{x}_{i+1}$ is the image of $x_{i+1}$ in $\bar{A}, \bar{I}_{1}=I_{1} \bar{A}, \ldots, \bar{I}_{s}=I_{s} \bar{A}$.

These elements satisfy the properties of filter-regular elements in [12] and elements of a complete reduction in [9]. That is why in [16] the author chose the name "(FC)".

The results of [16] showed that (FC)-sequences carry important information on mixed multiplicities. One of interesting results of [16] is the following theorem.

Theorem ([16, Theorem 4.1]). Let $A$ be a Noetherian local ring and $J$ an $\mathfrak{m}$ primary ideal. Let I be an ideal of positive height $h$. Suppose that $x_{1}, x_{2}, \ldots, x_{q}$ is a maximal (FC)-sequence in I with respect to $U=\{J, I\}$. Then

(i) $e\left(J^{[d-i]}, I^{[i]}\right)=e\left(J ; A /\left(x_{1}, x_{2}, \ldots, x_{i}\right)\right)$ for all $i \leq h-1$.

(ii) $e\left(J^{[d-i]}, I^{[i]}\right)=e\left(J ; A / \bigcup_{n \geq 0}\left[\left(x_{1}, x_{2}, \ldots, x_{i}\right): I^{n}\right]\right)$ for $h \leq i \leq q$.

(iii) $e\left(J^{[d-i]}, I^{[i]}\right) \neq 0$ if and only if $i \leq q$.

It follows readily from this theorem that for any $\mathfrak{m}$-primary ideal $J$, the length of maximal (FC)-sequences in $I$ with respect to $U=\{J, I\}$ is an invariant and this invariant is the largest nonvanishing degree of mixed multiplicities $e\left(J^{[d-i]}, I^{[i]}\right)$.

Based on the fact that maximal (FC)-sequences in $I$ with respect to $U$ have the same length (see Lemma 2.1(i)) and maximal sequences in $I$ satisfying conditions (i) and (ii) of (FC)-sequences have also the same length (see Proposition 2.2), we can state the first main result of this paper as follows:

Theorem 2.3. Let $J$ be $\mathfrak{m}$-primary. Let $I$ be an ideal of positive height ht $(I)$ and analytic spread $\ell(I)$. Suppose that $p$ is the length of maximal sequences in $I$ satisfying conditions (i) and (ii) of (FC)-sequences with respect to $U=\{J, I\}$ and $q$ is the length of maximal $(F C)$-sequences in $I$ with respect to $U$. Then

(i) $p=\operatorname{dim} R(I) / \bigcup_{n \geq 0}\left[J^{n+1} R(I): J^{n} R(I)\right]$.

(ii) $h t(I)-1 \leq q \leq p-1 \leq \ell(I)-1$.

(iii) Let $x_{1}, x_{2}, \ldots, x_{i}$ be a sequence in I satisfying conditions (i) and (ii) of (FC)sequences with respect to $U$, then $x_{1}, x_{2}, \ldots, x_{i}$ is an $(F C)$-sequence if and only if $i \leq q$.

At this point we remark that if $I$ is equimultiple, then as immediate consequences of Theorem 2.3 we have $h t(I)-1=q=p-1=\ell(I)-1$. Moreover, we also get 
some interesting results for mixed multiplicities and multiplicities of Rees rings of equimultiple ideals (see Theorem 3.1 and Theorem 3.2, Section 3).

Finally, we get the second main result of this paper as follows:

Theorem 3.3. Let $J$ be $\mathfrak{m}$-primary. Let $I$ be an ideal of positive height. Set $p=\operatorname{dim} R(I) / \bigcup_{n \geq 0}\left[J^{n+1} R(I): J^{n} R(I)\right]$. Suppose that $x_{1}, x_{2}, \ldots, x_{p}$ is a sequence in I satisfying conditions (i) and (ii) of (FC)-sequences with respect to $\{J, I\}$. Then $\left(x_{1}, x_{2}, \ldots, x_{p}\right)$ is a reduction of $I$.

\section{The Length of maXimal (FC)-Sequences}

From [16 Theorem 4.1] we see that the length of maximal (FC)-sequences is connected closely with the degrees of vanishing and non-vanishing of mixed multiplicities. In this section we give bounds for this length and characterizations for the length of maximal sequences satisfying conditions (i) and (ii) of (FC)-sequences.

We begin by establishing the following lemma.

Lemma 2.1. Let $J$ be an $\mathfrak{m}$-primary ideal and let $I$ be an ideal of positive height. Set $U=\{J, I\}$. Then

(i) Maximal (FC)-sequences in $I$ with respect to $U$ have the same length.

(ii) Let $e_{A}\left(J^{[d-i]}, I^{[i]}\right) \neq 0$ and $i>0$. Suppose that $x$ is an element in I satisfying conditions (i) and (ii) of (FC)-sequences with respect to $U$. Then $x$ also satisfies the condition (iii) of (FC)-sequences (i.e., $x$ satisfies the condition $(F C))$ with respect to $U$.

Proof. The proof of (i): By [16] Theorem 4.1], it follows that the length of any maximal (FC)-sequence in $I$ with respect to $U$ is equal to

$$
\max \left\{i \mid e_{A}\left(J^{[d-i]}, I^{[i]}\right) \neq 0\right\} .
$$

So the length of maximal (FC)-sequences in $I$ with respect to $U$ is an invariant.

The proof of (ii): Set $N:=\bigcup_{n \geq 0}\left(0: I^{n}\right) ; A^{*}=A / N, J^{*}=J A^{*}, I^{*}=I A^{*}$. We denote by $B(m, n)$ the Bhattacharya polynomial of function $l_{A^{*}}\left(\frac{J^{* m} I^{* n}}{J^{* m+1} I^{* n}}\right)$. By [2] we have $\operatorname{deg} B(m, n)=d-1$. Let $x \in I$ be an element satisfying conditions (i) and (ii) of (FC)-sequences with respect to $U$. If $x^{*}$ is the image of $x$ in $A^{*}$, then $x^{*}$ is not contained in any prime ideal belonging to Ass $A^{*}$ (see condition (ii) of (FC)). Hence $x^{*}$ is a non-zero-divisor in $A^{*}$. Set

$$
B=A^{*} / x^{*} A^{*}, \mathfrak{b}=J^{*} B, \mathfrak{c}=I^{*} B .
$$

For all large $m$ and $n$, we have

$$
\begin{aligned}
l_{B}\left(\frac{\mathfrak{b}^{m} \mathfrak{c}^{n}}{b^{m} \mathfrak{c}^{n}}\right) & =l_{A^{*}}\left(\frac{J^{* m} I^{* n}+\left(x^{*}\right)}{J^{* m+1} I^{* n}+\left(x^{*}\right)}\right)=l_{A^{*}}\left(\frac{J^{* m} I^{* n}}{J^{* m+1} I^{* n}+\left(x^{*}\right) \cap J^{* m} I^{* n}}\right) \\
& =l_{A^{*}}\left(\frac{J^{* n}}{J^{* m+1} I^{* n}+x^{*} J^{* m} I^{* n-1}}\right)(\text { see condition (i) of (FC)) } \\
& =l_{A^{*}}\left(\frac{J^{* m} I^{* n}}{J^{* m+1} I^{* n}}\right)-l_{A^{*}}\left(\frac{J^{* m+1} I^{* n}+x^{*} J^{* m} I^{* n-1}}{J^{* m+1} I^{* n}}\right) \\
& =l_{A^{*}}\left(\frac{J^{* m} I^{* n}}{J^{* m+1} I^{* n}}\right)-l_{A^{*}}\left(\frac{x^{*} J^{* m} I^{* n-1}}{J^{* m+1} I^{* n} \cap\left(x^{*}\right) \cap J^{* m} I^{* n}}\right) \\
& =l_{A^{*}}\left(\frac{J^{* m} I^{* n}}{J^{* m+1} I^{* n}}\right)-l_{A^{*}}\left(\frac{x^{*} J^{* m} I^{* n-1}}{x^{*} J^{* m+1} I^{* n-1}}\right) .
\end{aligned}
$$


Since $x^{*}$ is a non-zero-divisor in $A^{*}$, it follows that

$$
l_{A^{*}}\left(\frac{J^{* m} I^{* n-1}}{J^{* m+1} I^{* n-1}}\right)=l_{A^{*}}\left(\frac{x^{*} J^{* m} I^{* n-1}}{x^{*} J^{* m+1} I^{* n-1}}\right) .
$$

Hence

$$
\begin{aligned}
l_{B}\left(\frac{\mathfrak{b}^{m} \mathfrak{c}^{n}}{\mathfrak{b}^{m+1} \mathfrak{c}^{n}}\right) & =l_{A^{*}}\left(\frac{J^{* m} I^{* n}}{J^{* m+1} I^{* n}}\right)-l_{A^{*}}\left(\frac{J^{* m} I^{* n-1}}{J^{* m+1} I^{* n-1}}\right) \\
& =B(m, n)-B(m, n-1)
\end{aligned}
$$

for all large $m$ and $n$. By [16, Proposition 3.1] we have

$$
e_{A^{*}}\left(J^{*[d-i]}, I^{*[i]}\right)=e_{A}\left(J^{[d-i]}, I^{[i]}\right) .
$$

So $e_{A^{*}}\left(J^{*[d-i]}, I^{*[i]}\right) \neq 0$. Note that $i>0$. By $(*)$, it follows that $l_{B}\left(\frac{\mathfrak{b}^{m} \mathfrak{c}^{n}}{\mathfrak{b}^{m+1} \mathfrak{c}^{n}}\right)$ is a polynomial of degree $d-2$ for all large $m$ and $n$. Thus,

$$
\operatorname{dim} A / \bigcup_{n \geq 0}\left[(x): I^{n}\right]=d-1=\operatorname{dim} A^{*}-1
$$

and $x$ satisfies condition (iii) of (FC)-sequences.

By $(*)$, there exists a positive integer $z$ such that

$$
\begin{aligned}
l_{B}\left(\frac{\mathfrak{b}^{m} \mathfrak{c}^{n}}{\mathfrak{b}^{m+1} \mathfrak{c}^{n}}\right) & =l_{A^{*}}\left(\frac{J^{* m} I^{* n}}{J^{* m+1} I^{* n}}\right)-l_{A^{*}}\left(\frac{J^{* m} I^{* n-1}}{J^{* m+1} I^{* n-1}}\right) \\
& =B(m, n)-B(m, n-1)
\end{aligned}
$$

for all $m, n \geq z$. Fix an integer $v \geq z$, set $m=v$ and $Q(n)=B(v, n)$. Let $Q^{*}(n)$ denote the polynomial in $n$ of function $l_{B}\left(\frac{\mathfrak{b}^{v} \mathfrak{c}^{n}}{\mathfrak{b}^{v+1} \mathfrak{c}^{n}}\right)$. From this it follows that

$$
Q(n)-Q(n-1)=Q^{*}(n) .
$$

Motivated by this fact, we introduce the following result.

Proposition 2.2. Let $J$ be an $\mathfrak{m}$-primary ideal and let $I$ be an ideal of positive height. Set $U=\{J, I\}$. Then maximal sequences in I satisfying conditions (i) and (ii) of (FC)-sequences with respect to $U$ have the same length and this length is equal to $\operatorname{deg} Q(n)+1$.

Notes. (a) $J$ is an m-primary ideal, then $J I$ is nilpotent if and only if $I$ is nilpotent. (b) By [16, Remark 1], it follows that if $x_{1}, x_{2}, \ldots, x_{f}$ is a sequence in $I$ satisfying conditions (i) and (ii) of (FC)-sequences with respect to $\{J, I\}$, then $x_{1}, x_{2}, \ldots, x_{f}$ is a maximal sequence if and only if $\bar{I}=I\left[A /\left(x_{1}, x_{2}, \ldots, x_{f}\right)\right]$ is nilpotent.

Proof of Proposition 2.2. Since ht $(I)>0$, there exists an element $x_{1} \in I$ satisfying conditions (i) and (ii) of (FC)-sequences (see [16, Remark 1]). Let $x_{1}, x_{2}, \ldots, x_{p}$ be a maximal sequence in $I$ satisfying conditions (i) and (ii) of (FC)-sequences with respect to $U$. Set $t=\operatorname{deg} Q(n)$. Since $\operatorname{ht}(I)>0, \operatorname{deg} Q(n) \geq 0$. We shall begin by showing that $p=t+1$. Set $N:=\bigcup_{n>0}\left(0: I^{n}\right) ; A^{*}=A / N, J^{*}=J A^{*}, I^{*}=I A^{*}$. Let $x_{1}^{*}$ denote the image of $x_{1}$ in $A^{*}$. Set $B=A^{*} / x_{1}^{*} A^{*}, \mathfrak{b}=J^{*} B, \mathfrak{c}=I^{*} B$. We will denote by $x_{i}^{\prime}$ the image of $x_{i}$ in $B$ for $i=2, \ldots, t$. The proof is by induction on $t$. For $t=0$, by $(* *)$ we have $Q^{*}(n)=0$. From this it follows that $\mathfrak{b} \mathfrak{c}$ is nilpotent. Hence $\mathfrak{c}$ is nilpotent by Note (a). By Note (b), this implies $p=1$. So $p=\operatorname{deg} Q(n)+1$. Suppose that the result has been proved for $t-1 \geq 0$, we need to show that the result is true for $t$. Since $\operatorname{deg} Q^{*}(n)=t-1$ and $x_{2}^{\prime}, \ldots, x_{p}^{\prime}$ is also a maximal sequence 
in $\mathfrak{c}$ satisfying conditions (i) and (ii) of (FC)-sequences with respect to $\{\mathfrak{b}, \mathfrak{c}\}$. By inductive assumption, it follows that $p-1=\operatorname{deg} Q^{*}(n)+1=t$. Thus, $p=t+1$. The induction is complete. By the result just obtained, it follows that the length of maximal sequences in $I$ satisfying conditions (i) and (ii) of (FC)-sequences with respect to $U$ is an invariant and this invariant is equal to $\operatorname{deg} Q(n)+1$.

Let $I$ be an ideal of positive height. We call $\ell(I):=\operatorname{dim} \bigoplus_{n \geq 0}\left(I^{n} / \mathfrak{m} I^{n}\right)$ the analytic spread of $I$. Then our first main result is the following theorem.

Theorem 2.3. Let $J$ be $\mathfrak{m}$-primary. Let $I$ be an ideal of positive height $h t(I)$ and analytic spread $\ell(I)$. Suppose that $q$ is the length of maximal $(F C)$-sequences in $I$ with respect to $U=\{J, I\}$ and $p$ is the length of maximal sequences in I satisfying conditions (i) and (ii) of (FC)-sequences with respect to $U$. Then

(i) $p=\operatorname{dim} R(I) / \bigcup_{n \geq 0}\left[J^{n+1} R(I): J^{n} R(I)\right]$.

(ii) $h t(I)-1 \leq q \leq p-1 \leq \ell(I)-1$.

(iii) Let $x_{1}, x_{2}, \ldots, x_{i}$ be a sequence in I satisfying conditions (i) and (ii) of (FC)sequences with respect to $U$, then $x_{1}, x_{2}, \ldots, x_{i}$ is an $(F C)$-sequence if and only if $i \leq q$.

Proof. The proof of (i). Let $B(m, n)$ denote the Bhattacharya polynomial of function

$l_{A}\left(\frac{J^{m} I^{n}}{J^{m+1} I^{n}}\right)$. We assume that $B(m, n)=l_{A}\left(\frac{J^{m} I^{n}}{J^{m+1} I^{n}}\right)$ for all $m, n \geq z$. Fix an integer $v \geq z$, set $m=v$ and $Q(n)=B(v, n)$. Set

$$
M_{v}=\frac{J^{v} R(I)}{J^{v+1} R(I)},
$$

it is easily seen that $M_{v}$ is a finitely generated graded $R(I)$-module (see [4]). Evidently, the component of degree $n$ of $M_{v}$ is $\left(M_{v}\right)_{n}=J^{v} I^{n} / J^{v+1} I^{n}$. Consequently,

$$
l_{A}\left[\left(M_{v}\right)_{n}\right]=l_{A}\left(\frac{J^{v} I^{n}}{J^{v+1} I^{n}}\right)=Q(n)
$$

is a polynomial in $n$ having $\operatorname{degree} \operatorname{dim} M_{v}-1$ for all $n>v$. It can be verified that $\operatorname{dim} M_{v}=\operatorname{dim}\left[R(I) / J^{v+1} R(I): J^{v} R(I)\right]$. By Proposition 2.2 we have $p=$ $\operatorname{deg} Q(n)+1$. Hence

$$
p=\operatorname{dim}\left[R(I) / J^{v+1} R(I): J^{v} R(I)\right] \text { for all large } v .
$$

Since

$$
J^{t+1} R(I): J^{t} R(I) \subseteq J^{t+2} R(I): J^{t+1} R(I)
$$

for all $t, \operatorname{dim} R(I) / \bigcup_{n \geq 0}\left[J^{n+1} R(I): J^{n} R(I)\right]=\operatorname{dim}\left[R(I) / J^{v+1} R(I): J^{v} R(I)\right]$ for all large $v$. Thus, $p=\operatorname{dim} R(I) / \bigcup_{n \geq 0}\left[J^{n+1} R(I): J^{n} R(I)\right]$.

The proof of (ii): We write the terms of total degree $d-1$ in the Bhattacharya polynomial $B(m, n)$ of the Bhattcharya function $l_{A}\left(\frac{J^{m} I^{n}}{J^{m+1} I^{n}}\right)$ as

$$
\sum_{i=0}^{d-1} e_{A}\left(J^{[d-i]}, I^{[i]}\right) \frac{m^{d-i-1} n^{i}}{i !(d-i-1) !} .
$$

Since $\sqrt{J^{v+1} R(I)}=\sqrt{\mathfrak{m} R(I)}$, it follows that

$$
\operatorname{dim}\left[R(I) / J^{v+1} R(I)\right]=\operatorname{dim}[R(I) / \mathfrak{m} R(I)]=\ell(I) .
$$

Further, we have

$$
\operatorname{dim}\left[R(I) / J^{v+1} R(I): J^{v} R(I)\right] \leq \operatorname{dim}\left[R(I) / J^{v+1} R(I)\right] .
$$


Using the results just obtained we get $p \leq \ell(I)$. Since

$$
e\left(J^{[d-q]}, I^{[q]}\right) \neq 0 \text { and } e\left(J^{[d-i]}, I^{[i]}\right)=0
$$

for all $i>q$,

$$
q=\operatorname{deg} \sum_{i=0}^{d-1} e_{A}\left(J^{[d-i]}, I^{[i]}\right) \frac{v^{d-i-1} n^{i}}{i !(d-i-1) !} \leq \operatorname{deg} Q(n) .
$$

So we get $q \leq p-1=\operatorname{dim} R(I) / \bigcup_{n>0}\left[J^{n+1} R(I): J^{n} R(I)\right]-1 \leq \ell(I)-1$. By [16. Theorem 4.1] we have $q \geq \operatorname{ht}(I)-1$. Thus, (ii) of Theorem 2.3 have been proved.

The proof of (iii): We first will prove by induction on $q$ that if $i \leq q$ and $x_{1}, x_{2}, \ldots, x_{i}$ is a sequence in $I$ satisfying conditions (i) and (ii) of (FC)-sequences with respect to $U$, then $x_{1}, x_{2}, \ldots, x_{i}$ is also an (FC)-sequence. If $q=0$, in this case the result is trivial. For suppose the result has been proved for $q-1 \geq 0$. As the next step, we claim that the result is true for $q$. On the one hand $q>0$ and on the other hand $e\left(J^{[d-q]}, I^{[q]}\right) \neq 0$. By Lemma 2.1(ii), it follows that $x_{1}$ satisfies condition (iii) of (FC)-sequences with respect to $U$. Hence by [16] Proposition 3.3], we get $e\left(J^{[d-q]}, I^{[q]}\right)=e_{\bar{A}}\left(\bar{J}^{[d-q]}, \bar{I}^{[q-1]}\right)$, where $\bar{A}=A /\left(x_{1}\right)$ and $\bar{J}=J \bar{A}, \bar{I}=I \bar{A}$. From this equality we have $e_{\bar{A}}\left(\bar{J}^{[d-q]}, \bar{I}^{[q-1]}\right) \neq 0$. It can be verified that $q-1$ is the length of maximal (FC)-sequences in $\bar{I}$ with respect to $\{\bar{J}, \bar{I}\}$. Moreover, $\bar{x}_{2}, \ldots, \bar{x}_{i}$ is a sequence satisfying conditions (i) and (ii) of (FC)-sequences with respect to $\{\bar{J}, \bar{I}\}$, where $\bar{x}_{j}$ the image of $x_{j}$ in $\bar{A}, j=2, \ldots, i$. Since $i-1 \leq$ $q-1$, by our inductive assumption applied to $(q-1)$ that $\bar{x}_{2}, \ldots, \bar{x}_{i}$ is a sequence satisfying conditions of (FC)-sequences with respect to $\{\bar{J}, \bar{I}\}$. Since $x_{1}$ satisfies the condition (FC) with respect to $U$ and $\bar{x}_{2}, \bar{x}_{3}, \ldots, \bar{x}_{i}$ is a sequence satisfying conditions of (FC)-sequences with respect to $\{\bar{J}, \bar{I}\}$, it follows that $x_{1}, x_{2}, \ldots, x_{i}$ is a sequence satisfying conditions of (FC)-sequences with respect to $U$. The induction is complete. So we have proved that if $i \leq q$ and $x_{1}, x_{2}, \ldots, x_{i}$ is a sequence in $I$ satisfying conditions (i) and (ii) of (FC)-sequences with respect to $U$, then $x_{1}, x_{2}, \ldots, x_{i}$ is an (FC)-sequence. Since $q$ is the length of maximal (FC)-sequences in $I$ with respect to $U$. Hence, using the result just obtained we get (iii). Theorem 2.3 has been proved.

\section{Maximal (FC)-Sequences and Reductions}

First, we will give some results on (FC)-sequences and mixed multiplicities of equimultiple ideals.

It is well-known that if $\ell(I)$ is the analytic spread of $I$, then $\operatorname{ht}(I) \leq \ell(I)$. In the case $\operatorname{ht}(I)=\ell(I)$, the ideal $I$ is called equimultiple.

Let $J$ be $\mathfrak{m}$-primary. Let $I$ be an equimultiple ideal of positive height. If $q$ is the length of maximal (FC)-sequences in $I$ with respect to $U=\{J, I\}$ and $p$ is the length of maximal sequences in $I$ satisfying conditions (i) and (ii) of (FC)-sequences, then by Theorem 2.3 we have ht $(I)-1=q=p-1=\ell(I)-1$. As a consequence of Theorem 2.3 and [16, Theorem 4.1], we immediately get the following result.

Theorem 3.1. Let $J$ be $\mathfrak{m}$-primary. Let I be an equimultiple ideal of positive height $h t(I)=h$. Suppose that $q$ is the length of maximal $(F C)$-sequences in I with respect to $U=\{J, I\}$. Then

(i) $q=h t(I)-1$.

(ii) The length of maximal sequences in I satisfying conditions (i) and (ii) of $(F C)$-sequences with respect to $U$ is equal to $h$. 
(iii) $e\left(J^{[d-i]}, I^{[i]}\right)=0$ if and only if $i \geq h t(I)$.

(iv) If $x_{1}, x_{2}, \ldots, x_{h-1}$ is a sequence in I satisfying conditions (i) and (ii) of (FC)sequences with respect to $U$, then $x_{1}, x_{2}, \ldots, x_{h-1}$ is also an $(F C)$-sequence and $e\left(J^{[d-i]}, I^{[i]}\right)=e\left(J ; A /\left(x_{1}, x_{2}, \ldots, x_{i}\right)\right)$ for all $i \leq h-1$.

From Theorem 3.1(iv) and [16, Theorem 4.2] we immediately get the following theorem for multiplicities of Rees rings of equimultiple ideals.

Theorem 3.2. Let $R:=\bigoplus_{n>0} I^{n}$ be the Rees algebra of an equimultiple ideal $I$ of positive height $h t(I)=h$. Let $J$ be an $\mathfrak{m}$-primary ideal of A. Suppose that $x_{1}, x_{2}, \ldots, x_{h-1}$ is an arbitrary sequence in I satisfying conditions (i) and (ii) of $(F C)$-sequences with respect to $\{J, I\}$. Then

$$
e((J, I t) ; R)=\sum_{i=0}^{h-1} e\left(J ; A /\left(x_{1}, \ldots, x_{i}\right)\right) \text { and } e(R)=\sum_{i=0}^{h-1} e\left(A /\left(x_{1}, \ldots, x_{i}\right)\right) .
$$

An ideal $\mathfrak{I}$ is called a reduction of $I$ if $\mathfrak{I} \subseteq I$ and $I^{n+1}=\mathfrak{I} I^{n}$ for some nonnegative integer $n$.

We proceed now to establish the second main result of this paper as follows.

Theorem 3.3. Let $J$ be $\mathfrak{m}$-primary. Let $I$ be an ideal of positive height. Set $p=\operatorname{dim} R(I) / \bigcup_{n \geq 0}\left[J^{n+1} R(I): J^{n} R(I)\right]$. Suppose that $x_{1}, x_{2}, \ldots, x_{p}$ is a sequence in I satisfying conditions (i) and (ii) of (FC)-sequences with respect to $\{J, I\}$. Then $\left(x_{1}, x_{2}, \ldots, x_{p}\right)$ is a reduction of $I$.

Proof. Let $x_{1}, x_{2}, \ldots, x_{p}$ be a sequence in $I$ satisfying conditions (i) and (ii) of (FC)-sequences with respect to $\{J, I\}$. Since

$$
\operatorname{dim} R(I) / \bigcup_{n \geq 0}\left[J^{n+1} R(I): J^{n} R(I)\right]=p,
$$

$x_{1}, x_{2}, \ldots, x_{p}$ is a maximal sequence by Theorem 2.3(i). By Note (b), this implies that $I\left[A /\left(x_{1}, x_{2}, \ldots, x_{p}\right)\right]$ is nilpotent. Hence $\sqrt{I}=\sqrt{\left(x_{1}, x_{2}, \ldots, x_{p}\right)}$. Next, the proof is by induction on $i$ that $\left(x_{1}, x_{2}, \ldots, x_{i}\right) \cap I^{n+1}=\left(x_{1}, x_{2}, \ldots, x_{i}\right) I^{n}$ for all large $n$ and all $i \leq p$. If $i=0$, in this case the result is trivial. For suppose the result has been proved for $i-1 \geq 0$. As the next step, we claim that the result is true for $i$. Set $I^{\prime}:=\bigcup_{n \geq 0}\left[\left(x_{1}, x_{2}, \ldots, x_{i-1}\right): I^{n}\right]$. Denote by $x_{i}^{\prime}$ the image of $x_{i}$ in $A /\left(x_{1}, x_{2}, \ldots, x_{i-1}\right)$. Since $x_{i}^{\prime}$ is an element satisfying condition (i) of (FC)sequences, it can be verified that $\left(I^{n+1}+I^{\prime}\right) \cap\left(I^{\prime}, x_{i}\right)=\left(x_{i} I^{n}+I^{\prime}\right)$ for all large $n$. From this it follows that

$$
\begin{aligned}
I^{n+1} \cap\left(I^{\prime}, x_{i}\right) & =I^{n+1} \cap\left(I^{n+1}+I^{\prime}\right) \cap\left(I^{\prime}, x_{i}\right) \\
& =I^{n+1} \cap\left(x_{i} I^{n}+I^{\prime}\right)=x_{i} I^{n}+I^{\prime} \cap I^{n+1}
\end{aligned}
$$

for all large $n$. Therefore,

$$
I^{n+1} \cap\left(I^{\prime}, x_{i}\right)=x_{i} I^{n}+I^{\prime} \cap I^{n+1}
$$

for all large $n$. By the Artin-Rees lemma, then there exists an integer $t$ such that $I^{n} \cap I^{\prime}=I^{n-t}\left(I^{t} \cap I^{\prime}\right)$ for all $n \geq t$. Hence, as $I^{n} I^{\prime} \subseteq\left(x_{1}, x_{2}, \ldots, x_{i-1}\right)$ for large $n$, we get $I^{n} \cap I^{\prime}=I^{n-t}\left(I^{t} \cap I^{\prime}\right) \subseteq I^{n} \cap I^{n-t} I^{\prime} \subseteq I^{n} \cap\left(x_{1}, x_{2}, \ldots, x_{i-1}\right)$ for all large $n$. Consequently,

$$
I^{n} \cap I^{\prime}=I^{n} \cap\left(x_{1}, x_{2}, \ldots, x_{i-1}\right)
$$

for large $n$. By our inductive assumption applied to $(i-1)$ we get

$$
\left(x_{1}, x_{2}, \ldots, x_{i-1}\right) \cap I^{n+1}=\left(x_{1}, x_{2}, \ldots, x_{i-1}\right) I^{n}
$$


for all large $n$. Combining this fact with (2), we have $I^{n+1} \cap I^{\prime}=\left(x_{1}, x_{2}, \ldots, x_{i-1}\right) I^{n}$ for all large $n$. Hence by (1), we get $I^{n+1} \cap\left(I^{\prime}, x_{i}\right)=\left(x_{1}, x_{2}, \ldots, x_{i-1}\right) I^{n}+x_{i} I^{n}=$ $\left(x_{1}, x_{2}, \ldots, x_{i}\right) I^{n}$ for all large $n$. It follows readily from this that

$$
I^{n+1} \cap\left(x_{1}, x_{2}, \ldots, x_{i}\right)=\left(x_{1}, x_{2}, \ldots, x_{i}\right) I^{n}
$$

for all large $n$. The induction is complete. Since $\sqrt{I}=\sqrt{\left(x_{1}, x_{2}, \ldots, x_{p}\right)}$, it follows that $I^{n} \subseteq\left(x_{1}, x_{2}, \ldots, x_{p}\right)$ for all large $n$. Hence

$I^{n+1}=I^{n+1} \cap\left(x_{1}, x_{2}, \ldots, x_{p}\right)=\left(x_{1}, x_{2}, \ldots, x_{p}\right) I^{n}$ for all large $n$. Consequently, $\left(x_{1}, x_{2}, \ldots, x_{p}\right)$ is a reduction of $I$. The proof of Theorem 3.3 is complete.

Remark 3.4. Let $I, J, p$ and $x_{1}, x_{2}, \ldots, x_{p}$ as in Theorem 3.3. Then combining

(2) with (3), we get $I^{n+1} \cap\left(\bigcup_{j \geq 0}\left[\left(x_{1}, x_{2}, \ldots, x_{i}\right): I^{j}\right]\right)=\left(x_{1}, x_{2}, \ldots, x_{i}\right) I^{n}$ for all large $n$ and all $i \leq p$.

Remark 3.5. From Theorem 3.1(ii) and Theorem 3.3 it follows that if $I$ is an equimultiple ideal of positive height $h$ and $x_{1}, x_{2}, \ldots, x_{h}$ is a sequence in $I$ satisfying conditions (i) and (ii) of (FC)-sequences with respect to $\{J, I\}$, then $\left(x_{1}, x_{2}, \ldots, x_{h}\right)$ is a reduction of $I$.

\section{ACKNOWLEDGEMENT}

The author wishes to thank the editor for his help and encouragement. Special thanks are due to the referee for several constructive suggestions.

\section{REFERENCES}

1. A. Auslander and D. Buchsbaum, Codimension and multiplicity, Ann. Math. 68 (1958), 625657. MR 20:6414 MR 21:5658

2. P.B. Bhattacharya, The Hilbert function of two ideals, Proc. Cambridge Phil. Soc. 53(1957), 568-575. MR 19:727b

3. M. Herrmann, E. Hyry, J. Ribbe and Z. Tang, Reduction numbers and multiplicities of multigraded structures, J. Algebra 197, 311-341(1997). MR 98k:13006

4. D. Katz and J.K. Verma, Extended Rees algebras and mixed multiplicities, Math. Z. 202(1989), 111-128. MR 90i:13024

5. D. Katz and J.K. Verma, On the multiplicity of blow-ups associated to almost complete intersection space curves, Comm. Algebra 22(2)(1994), 721-734. MR 94k:13003

6. D. G. Northcott and D. Rees, Reduction of ideals in local rings, Proc. Cambridge Phil. Soc. 50 (1954), 145-158. MR 15:596a

7. D. Rees, a-transforms of local rings and a theorem on multiplicities of ideals, Proc. Cambridge Phil. Soc. 57(1961), 8-17. MR 22:9521

8. D. Rees, Hilbert functions and pseudorational local rings of dimension two, J. London Math. Soc. 24(1981), 467-479. MR 83d:13032

9. D. Rees, Generalizations of reductions and mixed multiplicities, J. London Math. Soc. 29(1984), 397-414. MR 86e:13023

10. I. Swanson, Mixed multiplicities, joint reductions and quasi-unmixed local rings, J. London Math. Soc. 48(1993), 1-14. MR 94d:13027

11. B. Teissier, Cycles èvanescents, sections planes, et conditions de Whitney, Singularities à Cargése, 1972. Astérisque 7-8(1973), 285-362. MR 51:10682

12. N.V. Trung, Reduction exponents and degree bound for the defining equation of graded rings, Proc. Amer. Math. Soc. 101(1987), 229-234.

13. J.K. Verma, Rees algebras and mixed multiplicities, Proc. Amer. Math. Soc. 104(1988), 10361044. MR 89d:13018

14. J. K. Verma, Rees algebras with minimal multiplicity, Comm. Algebra 17(12)(1988), 29993024. MR 91b:13032 
15. J. K. Verma, Multigraded Rees algebras and mixed multiplicities, J. Pure Appl. Algebra. 77(1992), 219-228. MR 93e:13005

16. D. Q. Viet, Mixed multiplicities of arbitrary ideals in local rings, Comm. Algebra 28(8)(2000), 3803-3821. MR 2001f: 13036

Department of Mathematics, Hanoi University of Technology, Dai Co Viet, Hanoi, VIETNAM

E-mail address: duongquocviet@bdvn.vnmail.vnd.net 